\title{
Aircraft Assembly Snags: Human Errors or Lack of Production Design?
}

\author{
Ageel Abdulaziz Alogla ${ }^{1, *}$ and Mansoor Alruqi ${ }^{2}$ (D) \\ 1 Department of Industrial Engineering, College of Engineering, Umm Al Qura University, \\ Mecca 21961, Saudi Arabia \\ 2 Department of Mechanical Engineering, College of Engineering, Shaqra University, \\ Ar Riyadh 11961, Saudi Arabia; malruqi@su.edu.sa \\ * Correspondence: aaogla@uqu.edu.sa
}

Citation: Alogla, A.A.; Alruqi, M. Aircraft Assembly Snags: Human Errors or Lack of Production Design? Aerospace 2021, 8, 391. https://doi.org/10.3390/ aerospace 8120391

Academic Editor: Daniel Ossmann

Received: 30 September 2021 Accepted: 3 December 2021 Published: 10 December 2021

Publisher's Note: MDPI stays neutral with regard to jurisdictional claims in published maps and institutional affiliations.

Copyright: (C) 2021 by the authors. Licensee MDPI, Basel, Switzerland. This article is an open access article distributed under the terms and conditions of the Creative Commons Attribution (CC BY) license (https:/ / creativecommons.org/licenses/by/ $4.0 /)$.

\begin{abstract}
To err is an intrinsic human trait, which means that human errors, at some point, are inevitable. Business improvement tools and practices neglect to deal with the root causes of human error; hence, they ignore certain design considerations that could possibly prevent or minimise such errors from occurring. Recognising this gap, this paper seeks to conceptualise a model that incorporates cognitive science literature based on a mistake-proofing concept, thereby offering a deeper, more profound level of human error analysis. An exploratory case study involving an aerospace assembly line was conducted to gain insights into the model developed. The findings of the case study revealed four different causes of human errors, as follows: (i) description similarity error, (ii) capture errors, (iii) memory lapse errors, and (iv) interruptions. Based on this analysis, errorproofing measures have been proposed accordingly. This paper lays the foundation for future work on the psychology behind human errors in the aerospace industry and highlights the importance of understanding human errors to avoid quality issues and rework in production settings, where labour input is of paramount importance.
\end{abstract}

Keywords: aerospace assembly; human error; poka-yoke; slips; mistakes; aircraft; lean manufacturing; zero defect

\section{Introduction}

Many manufacturing companies nowadays are shifting towards automating their production lines to avoid human errors [1,2]. Nevertheless, the level of adaption required to facilitate the different changes in each industry cannot be achieved without some necessary level of human intervention on the shop floor [3]. Humans are more adaptive, flexible, and creative than automated systems [4]. Various industries rely on and derive benefit from such human characteristics, enabling human hands to be involved in some complex assembly processes [5-7]. One major industry that heavily relies on human work is the aerospace industry [8,9]. Given the levels of human input involved in the processes in this sector, human errors are inevitable, and if they are not managed correctly, this might lead to potentially serious (even fatal) consequences, due to the critical nature of the aerospace industry [10]. Therefore, being able to identify and eliminate these errors at the source and redesign manufacturing systems by taking into consideration the nature of these errors represents an effective combination that can help mitigate, or even avoid, some of these errors.

The reality at present, however, is that human errors are typically confronted differently. Errors and snags, in general, can be confronted either reactively or proactively. Most industries have tried to establish such a proactive management culture that aims to proactively investigate and prevent problems at their sources. For the case of the aerospace industry, however, taking proactive measures is an obligatory part of the certification procedures regulated by the Federal Aviation Administration (FAA) [11]. In addition, the 
Aerospace Engine Supplier Quality Committee (AESQ), a group of giant aerospace businesses, bonded together to develop a standard approach for handling quality requirements, have emphasized the critical need to adopt prevention quality tools by the aerospace suppliers [12]. To comply with these regulations, players in the aerospace industry must commit to continuously improving their corrective and proactive measures.

Kolarik [13] describes the methods of meeting customer expectations, either by proactively focusing on process reliability and producing right-first-time products or setting up a final inspection zone, where specialists are responsible for preventing any non-conforming products from being passed on to the customer. In terms of this latter approach, various inspection practices are well-established, among which the acceptance sampling practice is considered to be the most efficient [14], despite its capability issues in inspection (i.e., calibration) [15].

From a lean perspective, Shingo (1986) believes that these types of sampling plans should not be followed, since they interrupt the production line. As a substitute, it has been argued that $100 \%$ in-line inspection for all units should be applied in a way that does not affect the pace of production. This implies using poka-yoke devices and sourcing the inspection either by self-checks or successive checks. The poka-yoke device concept has sparked the production of near-perfect products by Japanese factories with minimal inspection and rework [16]. In a similar vein, Monden [17] describes the inspection process as non-value-added activity and states that quality is the responsibility of each assembly cell owner, highlighting the importance of sourcing the quality in the process itself.

Inspection methods can be categorised into three main types: judgment, informative, and source inspection $[18,19]$. For judgment inspection, which needs to prevent the defective parts from reaching the customer, Shingo (1986) has described this type of inspection as being inefficient, since the products that have been produced with defects are already regarded as waste; therefore, stopping them from reaching the customer is costly and does not add value to the production process.

Informative inspection requires specific data to be collected from the shop floor and then seeks to understand and control the process in the future (e.g., statistical process control (SPC)). Shingo (1986) has added to this category with what he refers to as selfand successive checks, another two efficient means of informative inspection. Self-checks require each worker to individually inspect all products that are generated in each cell. The successive check, on the other hand, means that each operation worker should inspect the preceding work before starting their work. Although Shingo (1986) prefers self-checks to the successive check, Baudin [20] concludes that successive checks are preferred for assembly operations, as fitters may employ some bias, so as to deny any defects in their work. However, the decision of whether to self-check or not depends on the level of automation in the assembly line.

Finally, Shingo (1986) defines the third category, source inspection, as the process of determining any inappropriate settings before the process has started. He argues that each defect has its conditions and, thus, defect elimination can be achieved by chasing the related causes. Shingo (1986) asserts that by combining source inspection with either one of the two informative inspection types, defects can effectively be eliminated. To conclude, the mistake-proofing strategy is based on the fact that humans will inevitably make mistakes; therefore, if organisations seek to aspire toward a zero defects (ZD) approach, this necessitates integrating inspection as an internal part of the process itself (i.e., in-line inspection), rather than the traditional inspection techniques, where part specifications are inspected independently [18,21].

An appropriate overview of the different levels of quality controls, depending on the use of poka-yoke devices, can be illustrated in Table 1 below [22].

This paper contributes to the knowledge base by synthesizing the mistake-proofing literature with the literature of the psychology of human error and, based on this synthesis, develops a framework that helps lead towards obtaining deeper levels of insight into human error analysis. This developed framework is followed by a case study in the 
aerospace industry, in order to gain practical insights into the developed framework, offering a methodological investigation into the root causes of one of the snags raised in the aero-engine build line.

Table 1. The different inspection techniques for each quality level (adapted and modified from (Shimbun, 1989)).

\begin{tabular}{cc}
\hline The Quality Target & Type of Inspection Required \\
\hline Any generated defects can leave the factory & No inspection required \\
Generated defects do not leave the factory & Judgment inspection \\
Decreasing the defects & Informative inspection (e.g. SPC) \\
Defects do not leave the process & Self-check-in the process \\
Zero Defects (ZD) & Source inspection \\
\hline
\end{tabular}

This paper is structured as follows. Section 1 introduces the subject under study. Section 2 presents the developed framework that incorporates the psychology of human errors into mistake-proofing. Section 3 describes the case study conducted to obtain insights into the developed framework. Section 4 presents the findings obtained from the case study. Section 5 concludes this work and offers recommendations for future work.

\section{Developed Framework for Human Error Avoidance}

Deming [23] has highlighted that 95\% of defects are caused by system-related conditions, whereas only 5\% are linked to human-related errors. Consequently, designing prevention systems will lead to a decrease in the proportion of workers' errors. More specifically, all machines and processes can be reengineered or redesigned to increase the performance of a product. Humans, however, cannot be engineered to do so; instead, the proper approach should be to design the workplace, considering all physical and cognitive issues for the workers. Both humans and machines should be integrated, so that the overall performance of a product is increased [24].

As mentioned earlier in this paper, to err is human. This belief is commonly considered in the field of lean manufacturing literature and cognitive sciences. Nevertheless, most of the accessible lean manufacturing literature has concentrated on categorising pokayoke devices and giving numerous examples for these devices, without considering or explaining why these human mistakes occur. Stewart and Grout [24] state that mistakeproofing in the field of operations management needs a theoretical foundation that is concerned with and studies why humans make mistakes. This theoretical foundation has been established and guided to some extent in Stewart and Grout [24]'s work. The combination of cognitive science and mistake-proofing science has been discussed later on by the work of Pakdil et al. [25], where a methodology that combines human-related errors with system-related errors was proposed and followed by a case study to validate the model. Hence, it is crucial to psychologically understand and analyse human errors.

Norman [26] asks the question of why people stop investigating a problem once they find the person who made the error; while, in contrast, they continue investigating if they find that a machine has caused the defect. He adds that they will continue investigating mistakes until they find the root cause of the machine failure; on the other hand, they will start blaming and asking for more training once a human error has been identified. The author further clarifies by highlighting the widespread tendency to stop investigating the root cause once a worker's error is discovered. Kolarik [13] also supports this claim by stating that "finger-pointing" and "blame allocation", which is commonly known as a "blame culture", are not fruitful methods of reacting to the non-conformances caused by humans and other factors. In this culture, employers are not willing to take full responsibility for mistakes, in order to avoid criticism. On the other hand, the spread of what is known as "just a culture" in the aerospace industry, in which members believe that they can admit and address mistakes caused by systems without fear is preferable [27]. In this manner, employers are encouraged to learn from their mistakes/failures, even rewarded 
for reporting errors and incidents $[27,28]$. This is considered one of the main outputs of the just cultural environment, in which this act has a commitment for a working team to resiliently operate to meet the safety and quality requirements [29].

To have full control of risks, the aerospace industry has moved further to employ proactive practice by using safety management system (SMS) [30]. SMS is an organisational practice that focuses on four main areas: safety policies, risks management, safety assurance, and development of safety practice [30]. All of these practices are employed to maintain a certain level of product safety and meet the regulation requirements. Among these practices are the risks assessment and safety promotion. The prior practise promotes such procedures for identifying and assessing risks. The second practice encourages establishing a way of sustaining the safety assurance among products by continuous improvement and training.

To deploy SMS and address just culture, the root causes of the human error should be understood. Psychologists have attempted to understand how these errors occur. Moreover, they have tried to understand and define all the different mechanisms that lead the subconscious mind to make an error. They have also attempted to identify different factors that activate these mechanisms and, hence, prevent the occurrence of the error by isolating these factors, or at least considering them in the design phase. It is clear now how these studies can complementarily participate in achieving the goal of the mistake-proofing concept that Shingo (1986) has successfully described, where he mentions the technical aspect of this can be realised.

In terms of pioneering research in this field, Norman [31] and Reason and Mycielska [32] are regarded as being the most broadly acknowledged studies. These studies have differentiated between two different steps that lead to human error: (1) deciding a proper intention or plan and (2) reacting to that intention. Failure in deciding a proper intention or plan is called a mistake, while failure in following the procedures required to achieve this plan is called action slip. Each of these elements can be classified into different types, as will be demonstrated in the next section.

\subsection{Action Slips}

Action slips should form the focal point of any work that intends to investigate human errors, as this type of error is the base upon which Shingo [18] has established his concept of mistake-proofing. As presented in Table 2, Norman [31] discusses action slips, based on his theory of the activation-trigger-schemata system (ATS). This theory shows how activities that are frequently carried out will be stored as an unconscious routine known as schemas or schemata. Some of these schemas are triggered as needed when the conscious mind decides to perform a specific activity. For example, when a person wants to go to university by riding his motorcycle, a set of appropriate schemas are initiated. These schemas are classified into two main levels: high-level schemas, where the route to the university is found, and low-level schemas, such as motorcycle handling and balance, transportation rules, and crash avoidance. These schemas, in both high and low levels, stay and wait for any triggers or cues to activate. Once the person starts riding his motorcycle the "road finding" schema will be triggered as he reaches the first junction or roundabout. This will consequently trigger the other low schemas, such as following the motorcycle lane rules and steering/handling. These schemas are happening subconsciously, while it would be possible to listen to a broadcast via headphones that retains the conscious mind.

Norman [31] classifies the action slips based on the aforementioned levels of schemas. Moreover, he creatively identifies the sources where specific action slips occur. These sources are: slips in the formation of the intention caused by faulty activation of schemas and slips caused by a faulty triggering of these schemas. For the slips that occur during the formation of the intention, Norman (1981) identifies two types of error: (1) mode error, which results from inappropriately classifying the system status and (2) description error, where the target is unclearly specified to the worker. Mode errors occur when there is no clear picture of the status of the system. This usually happens when system designers want 
to use more than one command in one control device. For example, most digital watches have the characteristic of having more than one command on one side button. Description error occurs when there is a similarity in the specification of the target between two actions. A good example of this can occur when an intention is made to pour orange juice into a glass that is located beside a coffee cup. Instead of pouring the juice into the glass, the similarity of the coffee cup shape affects the intention, and a slip occurs, whereby the juice is poured into the coffee cup. For this type of slip, feedback is needed to give the user, or the fitter, in the case of this study, a clear status of the target $[26,31,33]$.

Table 2. Summary of the sources of the action slips, based on the activation-trigger-schemata system (ATS) (adapted from Norman (1981)).

\begin{tabular}{|c|c|c|c|}
\hline $\begin{array}{l}\text { Schemas } \\
\text { /Schemata }\end{array}$ & Slips & Types & Remarks \\
\hline \multirow{5}{*}{ High-level schema } & \multirow{2}{*}{$\begin{array}{l}\text { Slips in the formation of } \\
\text { the intention }\end{array}$} & Mode error & $\begin{array}{l}\text { Occurs when there is limited knowledge on the } \\
\text { targeted actions, resulting in improperly } \\
\text { classifying the system status. }\end{array}$ \\
\hline & & Description error & $\begin{array}{l}\text { Occurs where there is a similarity between } \\
\text { specifications of actions resulted in assigning the } \\
\text { incorrect specification to the targeted actions. }\end{array}$ \\
\hline & \multirow{3}{*}{$\begin{array}{l}\text { Faulty activation of } \\
\text { schemas }\end{array}$} & Capture error & $\begin{array}{c}\text { Occurs when a series of identical actions is } \\
\text { interrupted by a similar-looking but different } \\
\text { parametered action. }\end{array}$ \\
\hline & & Data-driven error & Initiated by an external stimulus or reason. \\
\hline & & Associative error & $\begin{array}{l}\text { Occurs when two separate activities with diverse } \\
\text { forms have a close interaction. }\end{array}$ \\
\hline Low-level schemas & $\begin{array}{l}\text { Faulty triggering of } \\
\text { active schemas }\end{array}$ & $\mathrm{n} / \mathrm{a}$ & $\begin{array}{l}\text { Occurs when two active schemas are active in } \\
\text { the subconscious mind at the same time, } \\
\text { producing a combination of acts or omissions to } \\
\text { one of them. }\end{array}$ \\
\hline
\end{tabular}

The second source of slips is faulty activation of schemas. This can occur either by unintentionally activating a schema or where activation of a schema is lost before its appropriate time is due. The former includes three main types which are: capture slips, datadriven slips, and associative slips. Capture slips occur when frequent sequenced identical actions are overlapped by an action that has a similar form but different parameter(s). Reason [34] clarifies this type of error using the example below:

"I meant to get my car out, but as I passed through the back porch on my way to the garage I stopped to put on my Wellington boots and gardening jacket as if to work in the garden".

In this example, the most frequent habit for this person, which is gardening, dominates his subconscious mind when that person wants to do new activities. This is because walking through the corridor is the same for gardening and going to the garage, but a different parameter (i.e., route) at the end of the corridor is needed. This source of error can be prevented by highlighting the critical point where the identical points overlap [33]. The data-driven slip is a type that is externally activated or triggered by an environmental cue or cause. Writing the word "Black" with a red colour and asking a person to quickly pronounce the printed colour is a good illustration of this type of slip. The last type of slip, associative slips, occurs when there is a strong link between two different actions that have different forms. This is beyond the scope of this project, as it usually occurs during speech, and no verbal commands are involved in the process of assembling the clips in this project.

On the other hand, fault activation can be raised when there is a loss of activation, caused by several reasons. These reasons are: the intention has been forgotten, recalling different elements, skipping procedures, or repeating procedures in the action sequence. 
Both Reason [35] and Norman [33] strongly described interruption as the main cause during the action sequence for these types of slips.

The last source of slips is a faulty triggering of active schemas. This happens when two active schemas are taking place in the subconscious mind simultaneously. Hence, a blend of actions or omissions to one of them is generated as a result. An example of blended actions occurring is when the intention is to say the word "close", while thinking of "shut", and one ends up saying "clut" [31]. Having two subconscious things to say or do is the main cause of this type of problem. Thus, multitasking is the key thing that should be avoided [26]. An example of one action being omitted would be when one thinks of ordering a coffee in a café, while typing some ideas into their laptop. After a while, the individual asks the coffee maker about their coffee order, but then only realises at that time that they have not even placed an order for it (i.e., omitted with competed schema, which is typing the ideas). Norman [26], in his later book, refers to this omission of action as a memory lapse.

\subsection{Mistakes}

As has been already discussed, mistakes are failures in decision-making. A noteworthy illustration of the different types of error involved in this domain can be found in the work of Norman [26]. These classifications are also compatible with Reason's descriptions in [35]. Mistakes are generally skill-, rule-, or knowledge-based errors. Most of the literature in this area refers to this type of error being commonly associated with novices and beginners, while action slips usually happen to experienced workers [26,33,35]. For this reason, this project will focus mainly on understanding how action slips occur on the shop floor, as the fitters being observed are sufficiently experienced and have received training. Figure 1 presents a framework that incorporates the various mechanisms behind human errors with the mistake-proofing concept.

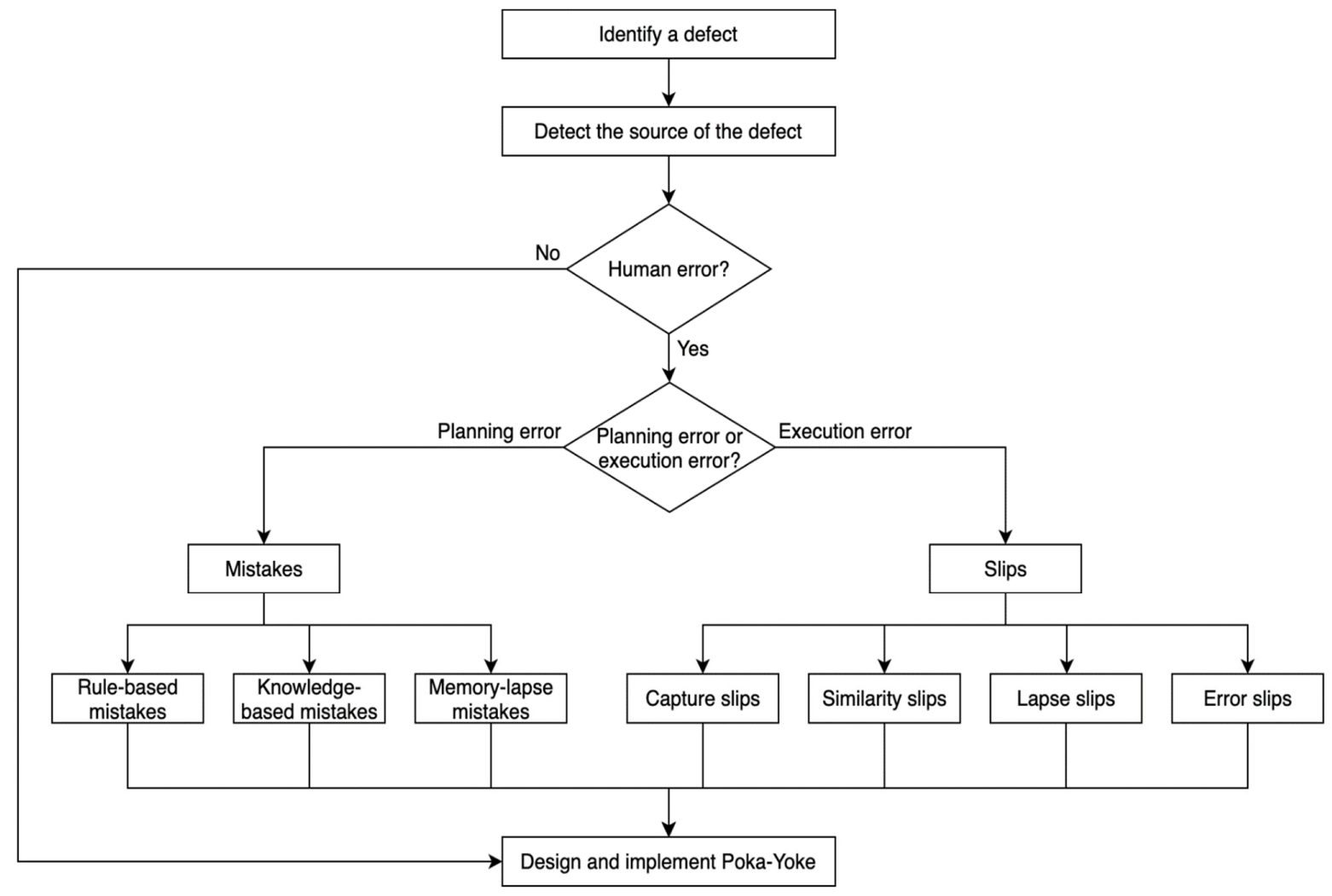

Figure 1. Framework designed to help identify and manage the root cause of aircraft snags (developed based on the work of Zhang [36] and Norman [26]). 


\section{Materials and Methods}

An exploratory approach has been adopted in this paper to help gather insights into the framework developed. A single case study was performed in a UK-based aerospace company. More specifically, this case study was conducted to discover the possible cause(s) of clip-related snags in the assembly process. Figure 2 presents the workflow for conducting the case study and validating the developed framework.

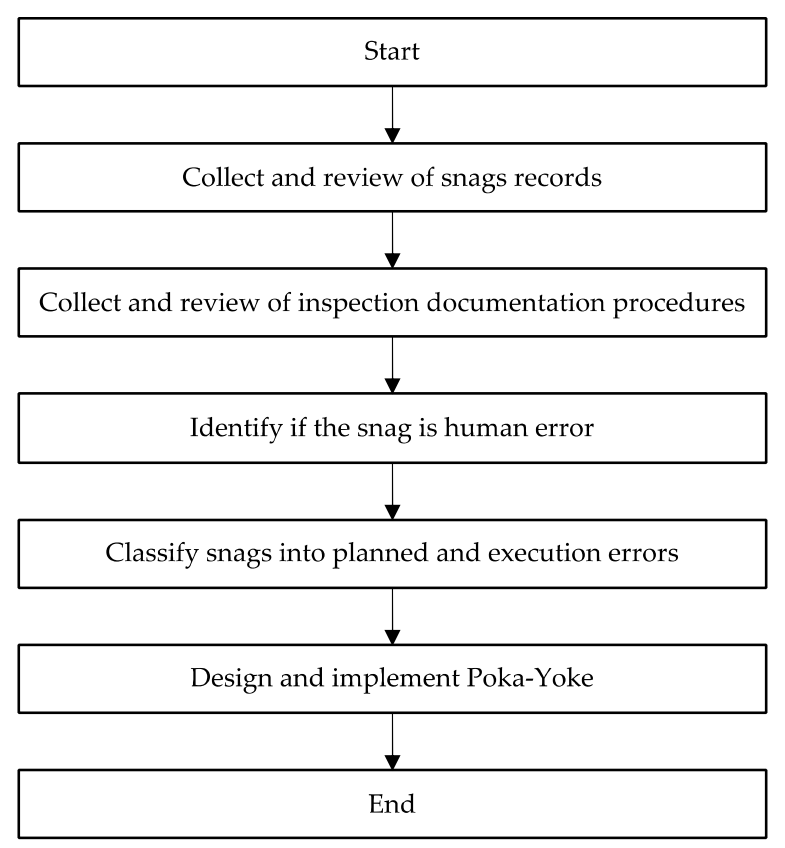

Figure 2. Workflow of the implementation of the proposed framework.

The build line at the host company refers to the area where all the different parts are assembled and from which the final part is dispatched. Following the assembly of harnesses and tubes in each one of these parts, each part is inspected before it is joined to the other parts. Should the inspector raise a snag, the fitter will come and rectify it, and another inspection will take place afterwards to double-check that the recent rectification conforms to the specifications. Following the combining and assembly of these parts, another inspection takes place, and the fitter should rectify any snag raised in this inspection as well. To assure that all engines conform to customer specifications, and to prevent any quality from escaping, a customer eye overcheck is undertaken, which is where the final inspection takes place.

Considerable snags are raised, due to issues with the clip-related assembly. These clips are used to externally assemble all electrical harnesses and tubes needed. Both the first and final inspectors visually and manually inspect the non-conforming assembled clips. Different descriptions were found to form these clip-related snags, and there was no clear answer as to what constituted the main reason(s) behind all these snags. Moreover, the case study revealed that the standard operating procedures (SOPs) for assembling these clips had not been reviewed to assure that they were understood and correctly followed.

As there was no clear indication of the main reason behind the clip-related snags, all of the different descriptions written and recorded by the inspectors in the inspection recording system over the past two years had been collected and categorised. The inspection recording system is a program that enables inspectors to record and describe all of the snags that are raised. In addition, a description of both the snag and the remedial action was obtained through this system.

The categories recorded were incorrect fitting, incorrect orientation, clip tightening loss, insulation, damage to the clip, and missing parts. According to the frequencies of 
each category, clip tightening loss accounted for the error with the highest number of frequencies (i.e., 33\% of clip-related snags). The inspection and subsequent rectification of this snag cost the host company a loss of $2.5 \mathrm{~h}$ a day, on average, over the past two years.

Following the collection and categorization of the recorded snags, a framework of the acceptable quality limits was identified by the first inspector, and the second/final inspector who check whether clips and harnesses assembly complied with standards. This means that such a guideline was proposed to identify the action slips by setting a practical reference that should identify the faulty actions. For example, both the first and final inspectors visually (i.e., by checking if there is a gap at the clip point) and manually (i.e., if the harness can be pulled through the clip) inspected the non-conforming assembled clips. A total of $65 \%$ of this type of snag was raised because a gap was found at the clip point, whereas the remaining proportion pertained to the harness being pulled through the clip. Therefore, the acceptable quality limits of the clip-related snags and a clear definition of the value were obtained using the documentation of these two inspection points.

In the beginning, process standards and procedures were checked and reviewed, and it was noted that they comply with the overall industry standards. According to the host company records, all fitters also received four-week training before commencing their career. In addition, there was no previous record of complaints or a lack of understanding in being able to correctly follow the procedures, which was indicative of a sufficient level of training for the fitters. Each wrench was calibrated and checked daily. This type of periodic calibration prevents wrenches from mechanical fatigue. There had also never been any reports of defective bolts; evidence of the host company's rigorous quality system for inspecting their supplies.

\section{Results and Discussion}

The observation of the fitters on the shop floor highlighted that the errors were attributed to human error, as fitters had simply forgotten to tighten the bolt. As the processes of assembling harnesses involved some of them to "temporary" tighten the bolt, so that the harness slack can be adjusted, the fitters sometimes forgot to readjust the clip and tighten the bolt completely. This may have been because the clip seemed fully tightened to the fitter. It should be noted that this step of "temporarily" tightening the bolt is written in the corresponding SOP document. Figures 3 and 4 provide some SOP examples that involve these steps for assembling the one component of the investigated part.

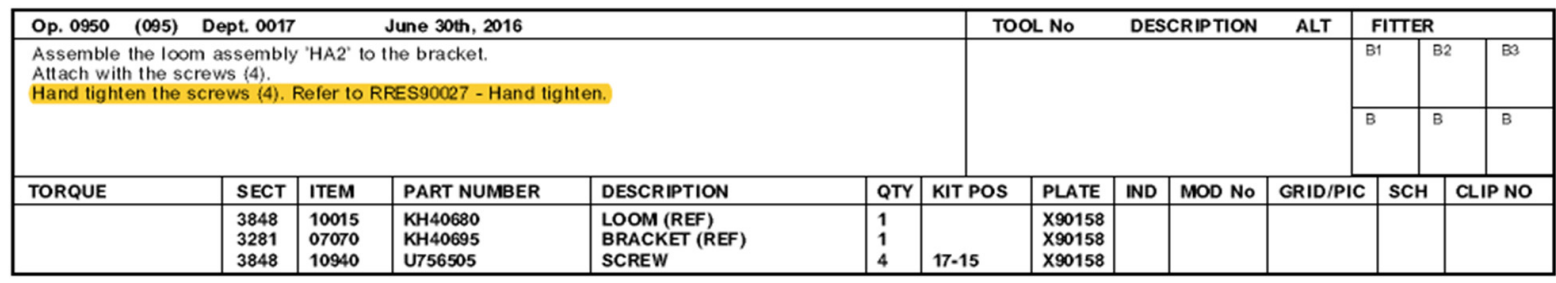

Figure 3. The SOP process confirming temporary tightening.

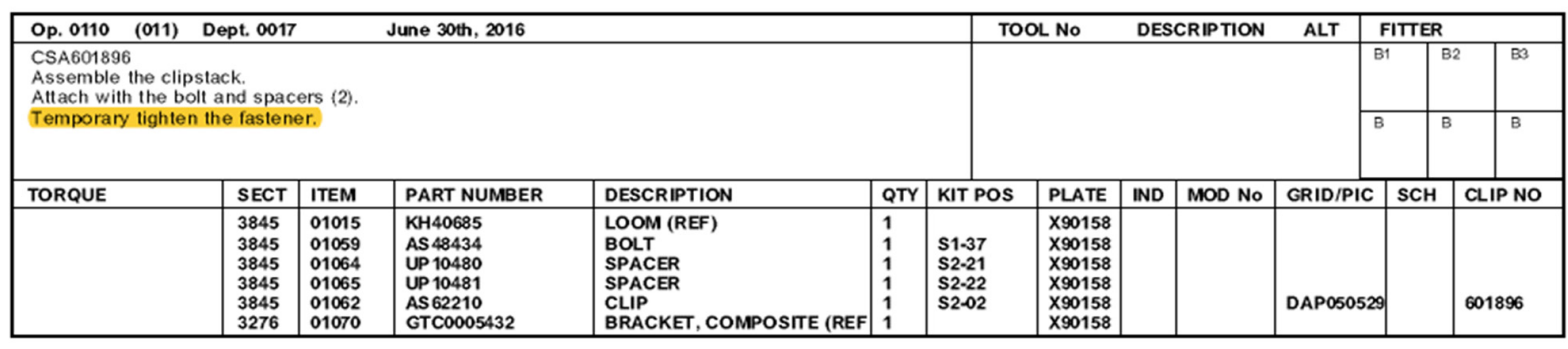

Figure 4. The SOP process confirming hand tightening of screws. 
The confusion of whether the bolt was fully tightened or temporarily tightened is caused by the difficulty in being able to distinguish between these two processes. Moreover, when the SOP states that a bolt should be retightened, the fitter sometimes thinks it has been already tightened, as the bolt takes place over the clip. Therefore, it is clear that this cause is critical and needs further consideration and investigation.

Missed tightening, which means forgetting to fully tighten the bolt, is due to human error. As discussed earlier, there are two types of human errors: mistakes and slips. Discussing the different forms of mistakes at this juncture would not be appropriate, since it has already been established that all fitters were experienced and sufficiently trained. Action slips occur mostly for experienced workers [26,33,35]; therefore, missed tightening can be analysed and understood from the perspective of action slips. Using the developed framework, presented in Figure 1, four different causes of missed tightening have been identified in this case study, as follows: (i) description similarity error, (ii) capture errors, (iii) memory lapses errors, and (iv) interruptions. Each of these is discussed below.

\subsection{Errors While Forming the Intention: Description Similarity Error}

In the error recognised as a description similarity slip, acting upon an element similar to the target is the reason for the fault. This occurs when the picture of the goal is insufficiently clear. In this case study scenario, the fitter was unable to distinguish between that which was fully (target) and temporarily tightened (similar to the target).

Therefore, a method of possibly distinguishing between the tightened and untightened bolt could be made with a visible mark or sign that provides a means of feedback to the fitter, as suggested in the host company SOPs. This concurs with what has been suggested in the literature, that description slips can be prevented by providing feedback to the fitter [26], which should reflect the status of the fitted bolt. The fitter cannot evaluate and compare the outcome of his/her action with the target unless he/she pulls the harness or checks the gap, which does not usually happen. Hence, in this instance, the poka-yoke device could be utilised to provide such feedback.

The wrench, shown in Figure 5, stamps a dark colour once the specified set torque is achieved. This provides visible feedback (i.e., marked with a colour, see Figure 6) to the fitters and can help them self-check and inspect their outcomes, which is the key element in Shingo's philosophy of quality control [18]. In other words, if any bolt has not been tightened, the fitter can swiftly observe the bolts that are not fully tightened, without the need for an inspector to come and check. This will source the inspection in the process itself, rather than waiting for another person to come and inspect the clip, which wastes time, effort, and money.

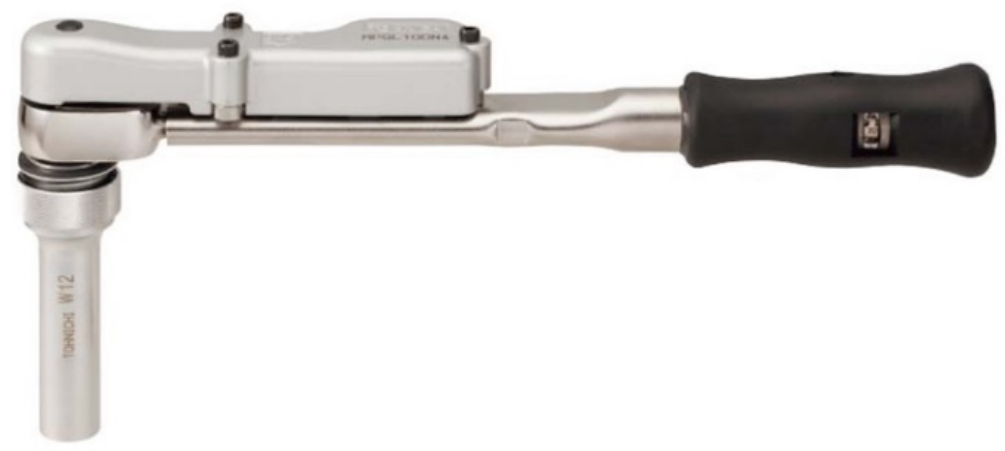

Figure 5. Error-proofing marking torque wrench. 


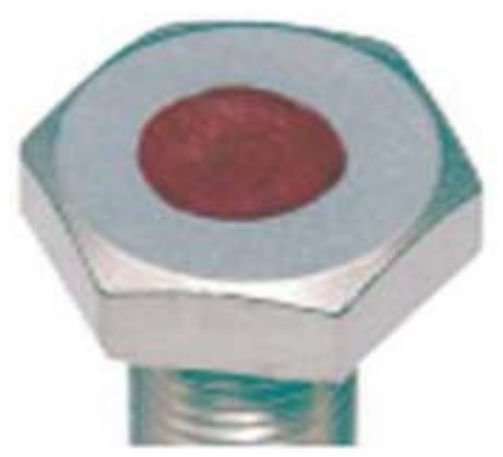

Figure 6. Marked bolt (red $\varphi 9)$.

\subsection{Errors Caused by Faulty Activation of Schemas: Capture Error}

As mentioned earlier, a capture error occurs when an action is frequently done and then another similar action, with different parameters, emerges and overlaps as a consecutive step. This leads to simply following the most recent frequent action parameters, instead of those that have emerged. The fitters on the shop floor are susceptible to such mistakes, as the SOPs do not consider this kind of error in their design. A simple example of an SOP that has such a design issue can be found in element A SOP pages, presented below in Figure 7.

\begin{tabular}{l}
\hline Op. 0160 (016) Dept. $0017 \quad$ June 30th, 2016 \\
\hline CSA602030 \\
Disassemble the clip stack. \\
Assemble the clipstack. \\
Torque the fastener to 47Ibf.in. Refer to RRES90027 - Class 1.
\end{tabular}

orque the fastener to $47 \mathrm{lb}$.in. Refer to RRES90027 - Class 1

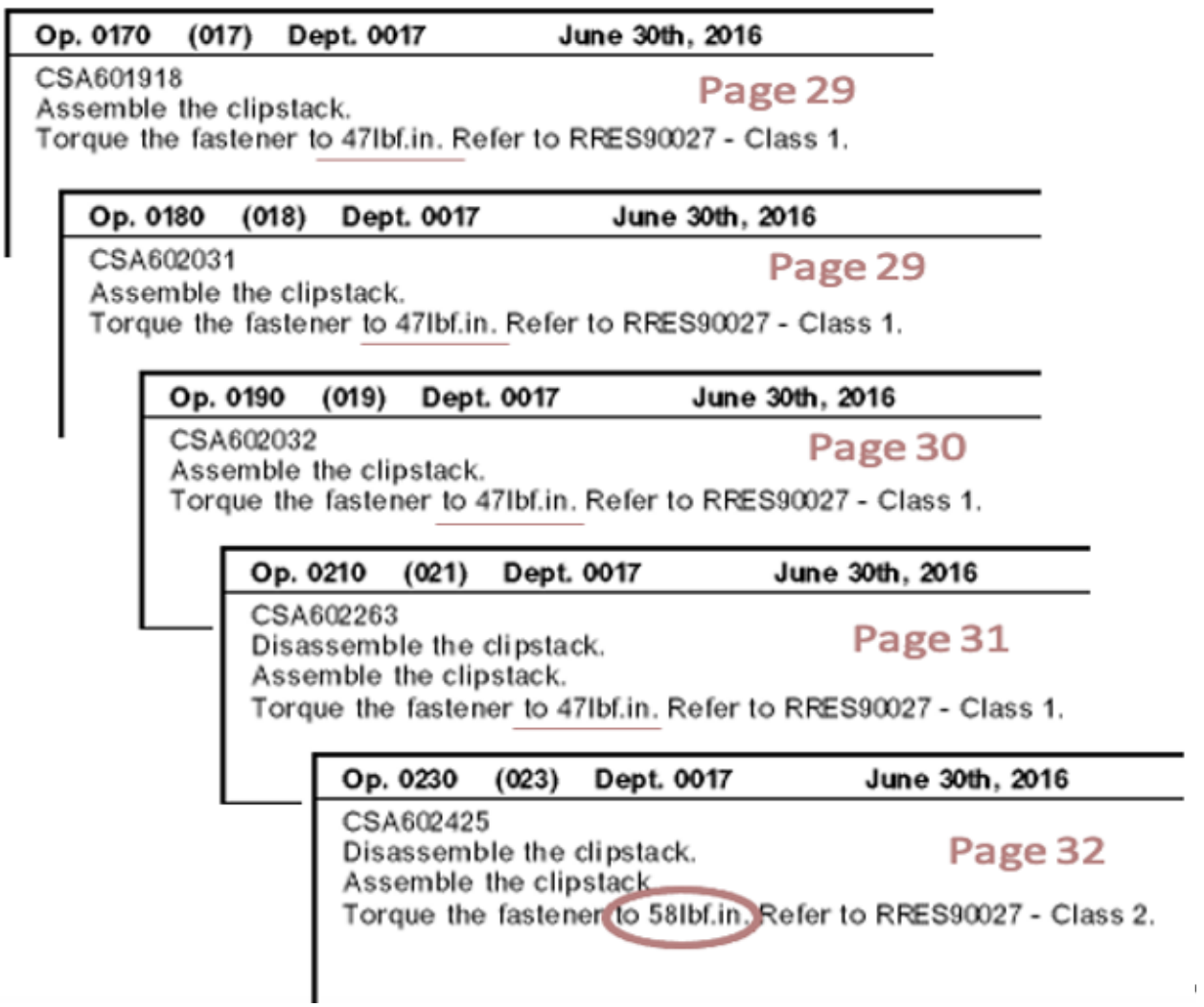

Figure 7. Successive SOPs overlap with similar action.

Based on these consecutive SOPs, highlighted in Figure 7, it is clear that an assembly procedure with a value of $47 \mathrm{Ibf}$.in is repeated from Pages 28-31, and then followed on Page 32 with a similar procedure that has a different value of 58Ibf.in. The fitter in this example 
might tend to complete the new procedure with the most frequent value that he/she had done before. In this case, he/she may stop torque tightening once a value of 47lbf.in is reached, since this was the most frequent and recent value that he/she had seen. Hence, this would result in a loss at the clip point, as the targeted value required/stipulated was not attained. This is only one case from several cases that have been found in different SOPs of the host company. It should be noted that experienced fitters are more likely to make such a mistake than novices. Intuitively, the more an individual repeats the processes involved in work, the more experienced he/she would become as a fitter. Thus, this kind of error is the result of repetitive or routine actions that manifest themselves as subconscious behaviour.

To avoid such a mistake from occurring in the future, a few solutions can be used. First, the designer, who is the planner, in this case, should ensure that there is no overlapping between any two identical forms of procedure. However, this is not always possible, due to the sequence of assembly required to achieve specific functions. A second, and more valid, solution would be to catch the critical point, where the most frequent action deviates and provide a clear, highlighted caution to the fitter for the new action. In this case, the colour of the new value (i.e., 58Ibf.in) can be changed or the style and format of the value can be altered (e.g., the value can be highlighted in bold). Other different highlighting ways can be used, as long as the critical point where the deviation occurs is clearly highlighted.

\subsection{Errors Caused by a Faulty Triggering of Two Active Schemas: Memory Lapse Slips}

This source of action slip usually results from activating two schemas simultaneously (e.g., multitasking). Doing two jobs at the same time is widely conceived as an efficient way of making progress. This kind of action slip occurred in the assembly line, where multitasking was found in a specific part of the clip assembly process. When an SOP states that a specific clip number is required, the fitter sometimes looks forward to the following step (mostly on the same page) to check the next clip number, so that he/she can get two clips in one go. Evidence of this can be found in Figure 8, where two different steps are contained on one page.

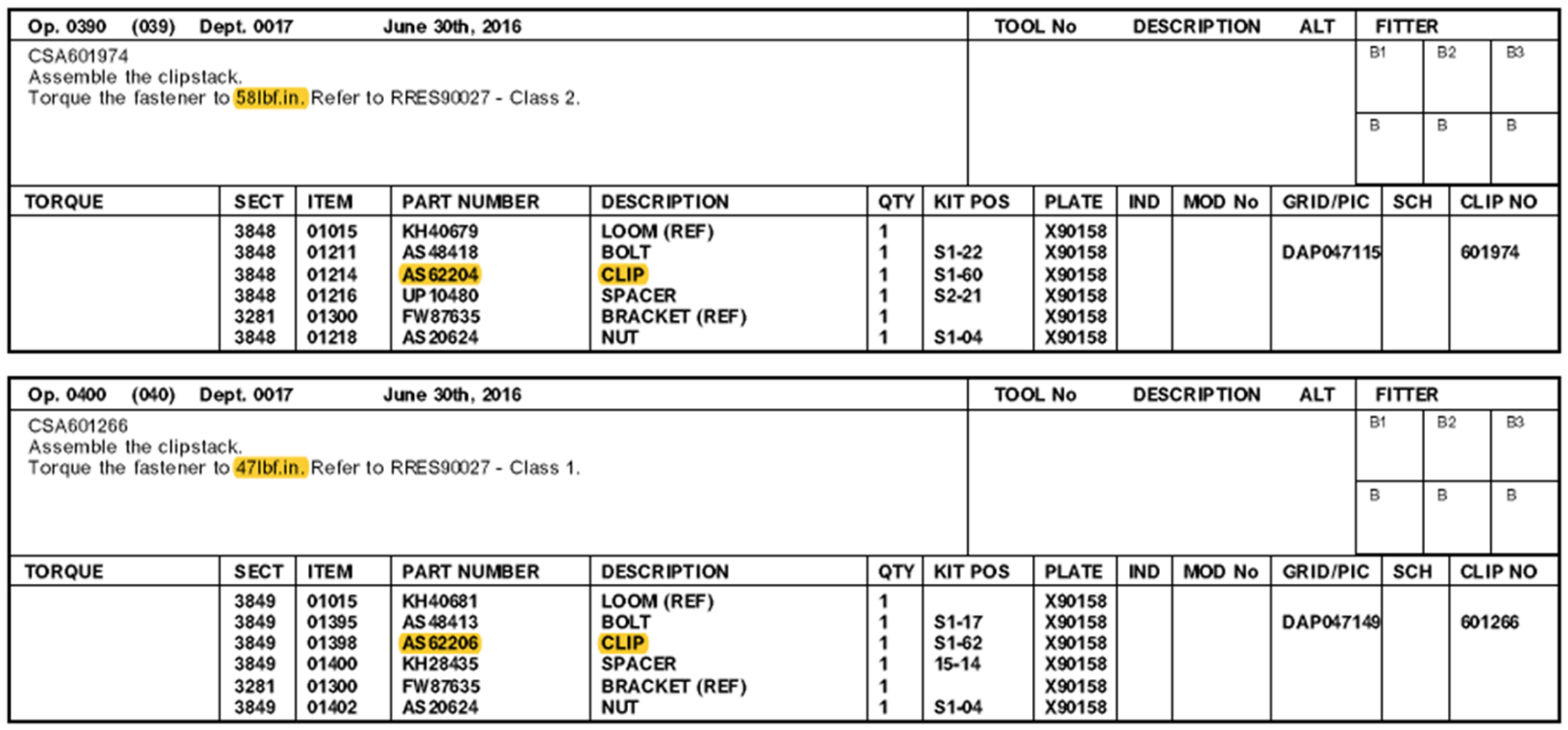

Figure 8. An SOP that consists of two different clip numbers.

What might happen is that the fitter will look at the first step and check which clip number is required. Following this, he/she will give the following step a quick look to get the next clip number, so that he/she can reduce his/her effort completing two clips in one attempt. Consequently, the fitter might subconsciously interchange these two clips while performing the task. This results in a loss at the clip point, since a smaller torque value was 
applied for a clip that was bigger than required (i.e., 47lbf.in applied for the clip number AS62204 in these two SOPs steps).

To prevent such action slips from occurring in the future, all related standards should point out that no more than one clip can be taken in each step in the relevant pages. In addition, memory lapses happen every day, due to the nature of human memory. Thus, to minimise the possibility of this type of error, as suggested by Norman [26], vivid reminders of steps are needed to complete the harness assembly. For instance, a vivid reminder should be written under each of the two steps, mentioned earlier, highlighting that no more than one clip should be taken in each step.

\subsection{A Common Cause of Action Slips: Interruption}

In addition to these three sources of action slips, interruption is another key slip that has been discussed by Norman [26], Norman [33], and Reason [35]. Observations of the assembly process revealed that with the cellular design of the assembly line, many visitors, seniors, and inspectors could easily interrupt fitters during their work. Each cell could be accessed easily, as there were no barriers or walls for those cells during work time. This does not mean that walls and doors should be placed around these cells, but the boundaries of the fitting area should be highlighted, so that any external person who wants to interrupt the fitters should determine when they will be free before accessing their workstations. Therefore, in the same manner, office workers sometimes require scheduling a meeting, and fitters in the build area should have the same or similar time management tool to reduce the instances of interruption.

However, this idea is not easy to implement, as fitters on the shop floor do not have the luxury or flexibility of being available at short notice in the same way that, perhaps, office workers do (e.g., half an hour). This is due to the need for maintaining an uninterrupted flow of actions and time frames, required to achieve assembly requirement deadlines. Moreover, fitters in the build line follow a sequence of procedures that are provided in the SOP and any interruption while performing these procedures might lead to loss of the original goal or intention. Fitters, therefore, need to have long and uninterrupted periods in the assembly line and a predetermined chunk of time for conducting necessary meetings with managers, planners, and inspectors. This chunk of time should be placed either before starting the assembly work or after finishing all the assembly work (e.g., $1 \mathrm{pm}-2 \mathrm{pm}$ for those workers who work from 6 am till $2 \mathrm{pm}$ ) or even clustering all meeting requests in one dedicated day. This can be implemented by the use of a programming language, so that all appointments can be clustered in a specific period of time.

\section{Conclusions}

In this paper, psychological mechanisms that might form human errors have been discussed. Understanding these psychological mechanisms can help lead towards obtaining deeper levels of insight on human error analysis. By merging streams of literature, this paper developed a framework that helps to identify the deeper causes of defects, considering the psychological mechanisms behind human error.

The methodology discussed in this work was applied to an exploratory case study from an aerospace company. The case study was undertaken to gain insights into the framework that was developed. The case study revealed that fitters are vulnerable to making different forms of errors that lead to them forgetting to fully tighten certain engine clip bolts. The first form of error is a description similarity error, which could be resolved as proposed in the literature and the company's SOPs, by using a mark to highlight whether the bolt is fully tightened or hand tightening is required. The poka-yoke device was proposed to eliminate this type of error. The second form of error highlighted was capture errors, where consecutive identical steps in the SOPs are commonly overlapped with similar steps that have different values. Highlighting the critical point where the overlapping occurs was suggested as a possible solution for this error. The last error was caused by the faulty triggering of two active schemas or multitasking, which was observed on the 
shop floor when the fitter took two different clips and inadvertently switched them over by mistake during the process. For this error, a standardization of single-tasking would need to take place in the SOPs. Moreover, it was also noted that, due to the cellular design of the build line, fitters could be easily interrupted, which may have been a contributing factor that led to some of these errors and, in particular, memory lapses. According to these results, human errors are inevitable, and the design of all of the components within the assembly system should be fitter-centric.

One limitation of this work is that the proposed solutions discussed, following the identification of the class of errors, might be of limited efficiencies, and other viable solutions might produce better results. It should also be noted that this study is exploratory and requires further investigations to evaluate the proposed framework and understand how human errors occur, as well as the various ways this framework can be employed to avoid these errors. Future work, therefore, requires the deployment of the proposed framework into multiple case studies, in order to ensure the effectiveness of the proposed framework. For scalability purposes, this framework could then be developed using one of the knowledge-based systems to provide a practical model that can be used in industry.

Author Contributions: Conceptualization, A.A.A.; methodology, A.A.A.; validation, A.A.A. and M.A.; formal analysis, A.A.A.; investigation, A.A.A.; resources, A.A.A. and M.A.; data curation, A.A.A. and M.A.; writing-original draft preparation, A.A.A.; writing-review and editing, M.A.; visualization. All authors have read and agreed to the published version of the manuscript.

Funding: This research received no external funding.

Conflicts of Interest: The authors declare no conflict of interest.

\section{References}

1. Doğru, A.; Bouarfa, S.; Arizar, R.; Aydoğan, R. Using convolutional neural networks to automate aircraft maintenance visual inspection. Aerospace 2020, 7, 171. [CrossRef]

2. Haight, J.M. Automated Control Systems Do They Reduce Human Error and Incidents? Prof. Saf. 2007, 52.

3. Pacaux-Lemoine, M.-P.; Trentesaux, D.; Rey, G.Z.; Millot, P. Designing intelligent manufacturing systems through Human-Machine Cooperation principles: A human-centered approach. Comput. Ind. Eng. 2017, 111, 581-595. [CrossRef]

4. Torres, Y.; Nadeau, S.; Landau, K. Classification and quantification of human error in manufacturing: A case study in complex manual assembly. Appl. Sci. 2021, 11, 749. [CrossRef]

5. Correia, D.; Silva, F.; Gouveia, R.; Pereira, T.; Ferreira, L.P. Improving manual assembly lines devoted to complex electronic devices by applying Lean tools. Procedia Manuf. 2018, 17, 663-671. [CrossRef]

6. Krugh, M.; Antani, K.; Mears, L.; Schulte, J. Prediction of defect propensity for the manual assembly of automotive electrical connectors. Procedia Manuf. 2016, 5, 144-157. [CrossRef]

7. Le, Y.; Qiang, S.; Liangfa, S. A novel method of analyzing quality defects due to human errors in engine assembly line. In Proceedings of the 2012 International Conference on Information Management, Innovation Management and Industrial Engineering, Sanya, China, 20-21 October 2012; pp. 154-157.

8. Beuß, F.; Sender, J.; Flügge, W. Ergonomics Simulation in Aircraft Manufacturing-Methods and Potentials. Procedia CIRP 2019, 81, 742-746. [CrossRef]

9. Judt, D.; Lawson, C.; Lockett, H. Experimental investigation into aircraft system manual assembly performance under varying structural component orientations. Proc. Proc. Inst. Mech. Eng. Part B J. Eng. Manuf. 2020, 234, 840-855. [CrossRef]

10. Aust, J.; Pons, D. Bowtie Methodology for Risk Analysis of Visual Borescope Inspection during Aircraft Engine Maintenance. Aerospace 2019, 6, 110. [CrossRef]

11. Federal Aviation Administration. Certification Procedures for Products and Articles. PART 21 2009. Available online: https: //www.ecfr.gov/current/title-14/chapter-I/subchapter-C/part-21 (accessed on 2 December 2021).

12. AESQ. How to Effectively Deploy Defect Prevention Methods in the Aero Engine Supply Chain; AESQ: Warrendale, PA, USA, 2019.

13. Kolarik, W.J. Creating Quality: Concepts, Systems, Strategies, and Tools; McGraw-Hill Inc.: New York, NY, USA, 1995.

14. Juran, J.M.; Gryna, F.M. Quality Control Handbook; McGraw Hill: New York, NY, USA, 1974.

15. Nof, S.Y.; Wilhelm, W.E.; Warnecke, H. Industrial Assembly; Springer Science \& Business Media: Hong Kong, China, 2012.

16. Womack, J.P.; Jones, D.T.; Roos, D. The Machine That Changed the World: The Story of Lean Production-Toyota's Secret Weapon in the Global Car Wars That is Now Revolutionizing World Industry; Simon and Schuster: New York, NY, USA, 2007.

17. Monden, Y. Toyota Production System. An Integrated Apprpach to Just-in-Time; Springer: Berlin/Heidelberg, Germany, 1983.

18. Shingo, S. Zero Quality Control: Source Inspection and the Poka-Yoke System; CRC Press: Tokyo, Japan, 1986.

19. Lazarevic, M.; Mandic, J.; Sremcev, N.; Vukelic, D.; Debevec, M. A systematic literature review of Poka-Yoke and novel approach to theoretical aspects. Stroj. Vestn. J. Mech. Eng. 2019, 65, 454-467. [CrossRef] 
20. Baudin, M. Lean Assembly: The Nuts and Bolts of Making Assembly Operations Flow; Productivity Press: Boca Raton, FL, USA, 2020.

21. Azamfirei, V. Robotic in-Line quality Inspection for Changeable Zero Defect Manufacturing; Mälardalen University: Västerås, Sweden, 2021.

22. Shimbun, N.K. Poka-Yoke: Improving Product Quality by Preventing Defects; CRC Press: Tokyo, Japan, 1989.

23. Deming, W.E. Out of the Crisis; MIT press: Cambridge, UK, 2018.

24. Stewart, D.M.; Grout, J.R. The human side of mistake-proofing. Prod. Oper. Manag. 2001, 10, 440-459. [CrossRef]

25. Pakdil, F.; Özkök, O.; Dengiz, B.; Kara, I.; Selvi, N.; Karg1, A. A systematic approach to reduce human and system-related errors causing customer dissatisfaction in a production environment. Total Qual. Manag. 2009, 20, 129-137. [CrossRef]

26. Norman, D. The Design of Everyday Things: Revised and Expanded Edition; Basic Books: New York, NY, USA, 2013.

27. Dekker, S.W.; Hugh, T.B. A just culture after Mid Staffordshire. BMJ Qual. Saf. 2014, 23, 356-358. [CrossRef] [PubMed]

28. Clare, J.; Kourousis, K.I. Analysis of Continuing Airworthiness Occurrences under the Prism of a Learning Framework. Aerospace 2021, 8, 41. [CrossRef]

29. Duffield, S.; Whitty, S.J. Developing a systemic lessons learned knowledge model for organisational learning through projects. Int. J. Proj. Manag. 2015, 33, 311-324. [CrossRef]

30. Wolf, $\mathrm{H}$. The emerging role of safety management systems in aerospace. In Proceedings of the 2012 IEEE Aerospace Conference, Big Sky, MT, USA, 3-10 March 2012; pp. 1-11.

31. Norman, D.A. Categorization of action slips. Psychol. Rev. 1981, 88, 1. [CrossRef]

32. Reason, J.T.; Mycielska, K. Absent-Minded?: The Psychology of Mental Lapses and Everyday Errors; Prentice Hall: Hoboken, NJ, USA, 1982.

33. Norman, D.A. Design rules based on analyses of human error. Commun. ACM 1983, 26, 254-258. [CrossRef]

34. Reason, J.T. Actions not as planned: The price of automatization. In Aspects of Consciousness; Underwood, G., Stevens, R., Eds.; Academic Press: Cambridge, MA, USA, 1979; pp. 1-67.

35. Reason, J. Human Error; Cambridge University Press: Cambridge, UK, 1990.

36. Zhang, A. Quality improvement through Poka-Yoke: From engineering design to information system design. Int. J. Six Sigma Compet. Advant. 2014, 8, 147-159. [CrossRef] 